1 Farrington T, Coward T, Onambele-Pearson G, Taylor RL, Earl P, Winwood K, 2016. An 2 investigation into the relationship between thickness variations and manufacturing 3 techniques of mouthguards. Dental Traumatology. 32(1):14-21

\title{
6 ABSTRACT.
}

8 Background: The aim of the present study was to measure the finished thickness of a

9 single identical $4 \mathrm{~mm}$ laminate mouthguard model from a large fabricated sample group

10 and to evaluate the degree of material thinning and variations during the fabrication

11 process.

12 Materials \& Methods: Twenty boxes were distributed to dental technicians, each

13 containing 5 duplicated dental models $(\mathrm{n}=100)$, alongside $5 \times 4 \mathrm{~mm}$ mouthguard blanks

14 and a questionnaire. The mouthguards were measured using electronic callipers

15 (resolution: $\pm 0.01 \mathrm{~mm}$ ) at three specific points. The five thickest and thinnest

16 mouthguards were examined using a CT scanner to describe the surface typography

17 unique to each mouthguard, highlighting dimensional thinning patterns during the

18 fabrication process.

19 Results: Of the three measurement sites, the anterior sulcus of the mouthguard showed 20 a significant degree of inconsistency (34\% coefficient of variation), in finished

21 mouthguard thickness between technicians. The mean thickness of the mouthguards in

22 the anterior region was $1.62 \pm 0.38 \mathrm{~mm}$ with a range of 0.77 to $2.80 \mathrm{~mm}$. This

23 inconsistency was also evident in the occlusion and posterior lingual regions but to a

24 lesser extent (12.2\% and 9.8\% variations respectively).

25 Conclusion: This study highlights variability in the finished thickness of the

26 mouthguards especially in the anterior region specific point, both within and between

27 individuals. At the anterior region measurement point of the mouthguard, the mean 
28 thickness was $1.62 \mathrm{~mm}$, equating to an overall material thinning of $59.5 \%$ when using a

29 single $4 \mathrm{~mm}$ laminate blank. 
32 Mouthguards are used as an intervention against trauma from violent impact between

33 the upper and lower dentition, transferring forces to the surrounding structures. A

34 mouthguard blank, when used for a sports mouthguard, is fabricated from a

35 predetermined thickness of material typically ranging between 3-6 $\mathrm{mm}$. Currently, there

36 is very little published information that is accessible to the public to make an informed

37 decision as to the correct thickness of mouthguard that should be worn for each type of

38 sport. The mouthguard is formed using a thermoforming process, where the

39 mouthguard material is heated between $80-120^{\circ} \mathrm{C}(1)$. This causes the ethylene vinyl

40 acetate (EVA) material to pass its glass transition temperature (Tg) of $84 \pm 3^{\circ} \mathrm{C}(2)$,

41 allowing the material to sag by 15 to $25 \mathrm{~mm}(3,4)$. After this phase it is then either

42 pulled down (in the case of vacuum forming) or forced down (in the case of the pressure

43 forming technique) over the dental model.

44 However, during the fabrication process there is an inherent further thinning of the

45 mouthguard material on heating and during the forming of the mouthguard (5). A

46 mouthguard's performance (i.e. energy absorbency) has been linked to the finished

47 postproduction thickness $(6,7)$. Therefore the greater the thickness of the finished

48 mouthguard the greater the ability to dissipate any impact force it may potentially

49 encounter. There are many production factors that could influence the degree of

50 thinning. For example, height and orientation of the model, duration of heating, degree

51 of material sag prior to forming, operator's level of experience, model size, palatal

52 depth, model position on platform, and model temperature $(4,8)$. Del Rossi and Leyte-

53 Vidal (5), examined the correlation between dental model height and the thinning of

54 mouthguard material at the anterior, canine, and molar sites. Their study found that

55 when using a $3 \mathrm{~mm}$ blank with a model height of $20 \mathrm{~mm}$ the material thinned to 1.6 
$56 \mathrm{~mm}$; thus equating to the material thinning by approximately $47 \%$. Similarly, at a model

57 height of $25 \mathrm{~mm}$, the material thinned to $1.4 \mathrm{~mm}$ (i.e. thinning by $53 \%$ ), and a model

58 height of $30 \mathrm{~mm}$ gave rise to material thinning to $1.2 \mathrm{~mm}$ (i.e. thinning by $60 \%$ ).

59 Interestingly, during all test conditions the molar cusp (occlusal) region thickness

60 remained constant at $1.6 \mathrm{~mm}$ (5). Geary and Kinirons (4) also investigated model height

61 in relation to material thinning. They found that by increasing the model height by

$6210 \mathrm{~mm}$ (from 25 to $35 \mathrm{~mm}$ ) this had a corresponding additional thinning of the EVA

63 material of $21 \%$ (from 1.53 to $1.21 \mathrm{~mm}$ ) when using a $3 \mathrm{~mm}$ blank. This gives an

64 overall thinning of the material from, in the case of the $25 \mathrm{~mm}$ model, a mouthguard

65 material thinning to $1.53 \mathrm{~mm}$ or by $49 \%$, and with the model at $35 \mathrm{~mm}$ a material

66 thinning of $1.21 \mathrm{~mm}$ or by $60 \%$ (4). Both Geary et al. (4) and Del Rossi et al. (5)

67 concluded that by keeping the dental model height low, the degree of material stretching

68 observed during the thermoforming process is minimized.

69 Geary et al. (4) investigated how prolonging the heating interval of the EVA material

70 prior to forming affected the finished mouthguard thickness. They found increasing the

71 duration of heating by 30 seconds actually decreased the amount of thinning in the

72 material. Initially this seems counter-intuitive, however, they postulated an explanation

73 relating to the proximity of the sagging EVA material with the dental model, whereby

74 the sagging EVA material contacts the model, transforming from its elastic plasticised

75 state to its plastic state, prior to the pressure being introduced (4). They also altered the

76 model position on the platform from the centre $(1.53 \mathrm{~mm})$, to the distal edge of the

77 model placed at the edge of the platform, finding that this significantly $(\mathrm{p}<0.01)$

78 increased the stretching of the material $(1.31 \mathrm{~mm})(4)$. 
80 Mizuhashi et al (9) examined the thickness and fit of a $3.8 \mathrm{~mm}$ blank during two

81 different thermoforming conditions. The conditions being (i) sheet lowered over the

82 model when vacuum applied and (ii) sheet lowered over the model prior to vacuum

83 applied. They measured anatomical points at both the incisal and first molar region and

84 found that there were differences in thickness between anatomical points. However,

85 there were no significant differences between thickness and condition. The thinning

86 patterns observed within these conditions were 40-42\% (incisal region), $32 \%$ (molar

87 region) and $23-24 \%$ occlusal region. They also found that the fit differed between the

88 two conditions. Mizuhashi et al. (10) also examined four heating conditions in relation

89 to thickness and fit, they found again that there was a difference in fit, which was

90 dependant on the heating method, but no difference was reported between method and

91 thickness between conditions. Thinning reported within this study ranged from $26-45 \%$

92 and was dependent upon the anatomical site. A study by Takahashi et al. (11) examined

93 the effects of six conditions, which varied in relation to height of the model and heating

94 procedures they reported that within conditions there was up to a $26 \%$ variation in

95 thickness difference. Holding conditions of the mouthguard blank during the heating

96 process has also been investigated, and this has been demonstrated to have an increase

97 within thickness (not sure I understand within thickness) of the processed material

98 especially when the mouthguard material is held at four points during heating (12).

99 Thus, from the mentioned literature it shows that technique plays an important crucial

100 factor within the fabrication process.

101 Previous studies have used callipers to record measurements. For instance, Geary et al.

102 (4) sectioned their mouthguard samples and measured at 12 points using a digital 103 micrometer (resolution $0.001 \mathrm{~mm}$ ). Del Rossi et al. (5) used a spring-loaded calliper 104 gauge (resolution $0.01 \mathrm{~mm}$ ) and measured the mouthguard thickness occlusally at each 
105 cusp of the first molars and labial from the central incisors and both right and left 106 canines, with an average mouthguard thickness value assigned to each region. In the 107 present study, measurement reference points were selected in the anterior sulcus, 108 posterior lingual section and occlusal measurements as shown in Table 1. Hence, to 109 develop our understanding of the factors that influence the finished mouthguard and 110 render these more reliable, the primary focus of this study was to examine the

111 reproducibility of the thermoforming task, describing the degree of both intra and inter-

112 individuals variability from a large cohort of commercial operators under normal

113 laboratory conditions (i.e. no experimental control of usual practice). Our objective was 114 to highlight how the reproducibility of the thermoforming task fared in relation to 115 mouthguard thickness and production consistency. 


\section{MATERIALS \& METHODS.}

119 Ethical approval was sought and obtained, prior to commencement of the study taking 120 place, from the ethics committee at the Department of Exercise and Sport Science,

121 Manchester Metropolitan University.

\section{Fabrication of Mouthguards.}

124 A total of 22 boxes were distributed to dental technicians, each containing 5 identical 125 duplicated dental models ( $\mathrm{n}=100$ models in total) and $5 \times 4 \mathrm{~mm}, \mathrm{EVA}, 120 \mathrm{~mm} \varnothing$ 126 (diameter), clear mouthguard blanks (Bracon Dental Laboratory Products, East Sussex, 127 UK). The rationale for the selection of the mouthguard material for the study was 128 linked to the fact that: EVA has been recorded as the most commonly used mouthguard 129 material $(8,13,14)$. Personal communications with material suppliers and internet 130 resources, indicated that $4-6 \mathrm{~mm}$ mouthguard blanks were the most common thickness

131 used in the construction of mouthguards for the majority of sports within the UK.

132 Each technician received a Participant Information Sheet that explained the blind study,

133 and they then completed the mouthguard production process in their usual manner and

134 returned the box if they were willing to partake. They were also asked to complete a

135 short questionnaire, which encompassed their level of experience (i.e. years in this 136 career), the type and age of their laboratory's mouthguard formation machine, the size

137 of blanks used, and any further details on the technique they routinely employed in 138 manufacturing mouthguards. All the questionnaires and mouthguards were analysed 139 and measured blindly.

\section{Measurements of the processed mouthguards.}


142 Following the return of the mouthguards, each box was assigned a code and each model

143 in the assigned box was also given a numerical number for reference purposes.

144 Anatomical plot points were marked on the master model, which indicated where all the

145 subsequent mouthguards were to be measured. These plot points were then transposed

146 onto the mouthguard using a permanent medium tipped marker pen, to ensure

147 consistency in the testing methodology. Three anatomical measurement points were

148 selected and marked on the finished mouthguards (Table $1 \&$ Figure 1), allowing for

149 precise comparisons to be made both within and between technicians.

150

$<$ INSERT TABLE 1 HERE $>$

152

$<$ INSERT FIGURE 1 HERE $>$

154

155 An electronic calliper gauge (External Digital Calliper 442-01DC Series, Moore \&

156 Wright, UK) was used to measure the thickness of the finished mouthguards. This type

157 of gauge, was chosen for ease and level of range of action, giving viable access to the

158 occlusal cusp areas of the mouthguard. The callipers had a range resolution of $159 \pm 0.01 \mathrm{~mm}$. Each mouthguard anatomical point was measured three times and a mean

160 value obtained, after each measurement the gauge was zeroed. It should be noted that

161 the callipers were calibrated using a $4 \mathrm{~mm}$ steel calibration block, grade 1, ISO-DIN-BS

162 (Cen Dev $\mu \mathrm{m}+0.02$, Max Dev +0.02, Min Dev -0.11, Variation 0.13; Alan Browne

163 Gauges Ltd, Leamington Spa, UK) and were frequently used to check the accuracy of

164 the gauges between the measurements sessions.

165

166 CT scanned comparison of two surfaces for model accuracy and the thickness of the

\section{7 finished mouthguards.}


168 Five master models and five randomly selected duplicate models were taken from the 169110 models prepared in the course of this study. These were then analysed using a 170 Computed tomography (CT) scanner (Scanner: GE Medical Systems) with the

171 following settings: - Light Speed 16, Mode of Capture - Helical, Gantry Tilt - 0 Voxel 172 Size $-0.7031 \times 0.7031 \times 0.5$, Matrix Size $-256 \times 256, \mathrm{KV}-120, \mathrm{Ma}-90$

173 Reconstructed in $0.625 \mathrm{~mm}$ axial slices. These scans would help to systematically 174 determine any degree of error between the models that could have occurred during the 175 replication process (Figure 2).

176 The computer software programme, Robin's 3D - 3D Editor software (V3.1.0.0)

177 (www.robins3d.co.uk), used an established algorithm technique to calculate the least square fit 178 points between the two images surfaces (15). Essentially, the programme fits the two images as 179 closely as possible to an average number of points (200) with the difference between the two 180 surfaces viewed as a colour that was assigned a numerical value that was set at $0.001 \mathrm{~mm}$. A 181 cursor was also placed on the surface to further confirm the difference between the surfaces.

182 There was a slight distortion as expected in the production of the duplicate models used for this

183 study; a $+/-0.2 \mathrm{~mm}$ discrepancy between the duplicated models was observed in the anatomical 184 region from where the thickness measurements were taken (Figure 2) which is deemed to be 185 within acceptable tolerances within dentistry (16). The five thickest and thinnest mouthguards 186 were also subjected to further analyses using a CT scanner using the scanning procedures 187 described above. 
193 Statistical analyses were performed using PASW ${ }^{\circledR}$ Statistics 18 (SPSS Inc., Chicago, IL, 194 USA). Parametricity checks were carried out using the Kolmogorov-Smirnov (for 195 normal distribution) and Levene's (for equal variance) tests. The statistical analyses to 196 identify the variability in mouthguard characteristics within technician groups were 197 tested through computing the Coefficients of Variation and Intraclass Correlation 198 Coefficients (2-way random model, absolute agreement). Between technicians/groups 199 differences were tested using factorial ANOVA (with appropriate post-hoc Independent 200 Bonferonni corrected 2-tailed $t$-tests). Where data did not obey the parametric 201 assumption, Kruskal Wallis analyses (with appropriate post-hoc Mann Whitney pairwise 202 comparisons) were run. The degree of association between dependent and independent 203 pairs of variables was investigated using correlations (Pearson or Spearman's204 depending on whether the data set was parametric or not). Data are presented as Mean \pm 205 STDEV, with the alpha set at $\leq 0.05$. 
206 RESULTS

\section{Questionnaire Results.}

208 The questionnaire-based survey from the cohort of technicians showed that $70 \%$ of the 209 participants generally used $4 \mathrm{~mm}$ blanks for their mouthguards, $25 \%$ used $3 \mathrm{~mm}$, and

210 only 5\% used $5 \mathrm{~mm}$ blanks (which they may have used for either single or dual

211 laminate). However, the vast majority (i.e. 75\%) of the participants did not laminate

212 mouthguard material to increase the finished thickness of the mouthguard. In total $90 \%$

213 used pressure forming machines to make their mouthguards. Furthermore, $70 \%$ of the

214 respondents had 20 years or more experience as technicians. The age of the

215 thermoforming machines ranged from 1 - to 20 years with a mean age of 6.6 years.

216 Research Question: How consistent were the technicians at the task of forming

217 mouthguards within and between groups at three anatomical measurement points?

218 A total of 20 of the 22 boxes were returned completed, which equates to a response rate 219 of $91 \%$.

222 When setting a threshold of 5\% for maximal acceptable coefficients of variation in

223 repeated mouthguard manufacture thicknesses, (see data in Figure 3), it was observed 224 that at Site A, all the technicians (to a lesser extent technicians 8, 10 \& 13) showed a 225 significant degree of inconsistency, with CV's reaching up to $34 \%$. At site B, Figure 3, 226 technicians 4, 6, 9-11, 13-17, 19-20; and Site C, Figure 3, technicians 1-3, 6, 9, 10, 14, 227 16-20; also showed significant inconsistencies in manufacturing thicknesses, though 228 inconsistencies here were less pronounced than those seen at Site A, reaching $12.2 \%$ in 229 Site B and $9.8 \%$ in site $\mathrm{C}$ respectively. 
230 A Kruskal Wallis test was used to compare the mean mouthguard thickness difference

231 observed between technicians; this showed that there was a significant technician effect

$232(\mathrm{p}<0.001)$ at all three sites.

233 Research Question: Did any of the following variables i.e. type and age of

234 thermoforming machine or level of experience of the operator/technician have a

235 significant influence on the finished thickness of the mouthguards?

236 It was shown that the make/model of the moulding machine and the average

237 mouthguard thickness were not significantly associated, regardless of the anatomical

238 site $(A-C)$ under consideration $(p>0.05)$. Similarly, there was no significant

239 correlation between the approximate age of the forming machine and the finished

240 thickness of the mouthguard $(\mathrm{p}>0.05)$. Also, there was no significant correlation

241 between the number of years' experience and the finished thickness of the mouthguard.

242 There was a statistically significant difference in mouthguard thickness within, and

243 between, sub samples/groups of participating technicians at the assigned measurement

244 points as shown in Figures 4-6. This is observed to a greater extent in the anterior

245 region (Figure 4) where the greatest degree of material stretching/thinning was noted.

\section{$<$ INSERT FIGURES 4-6 HERE >}

248 Some showed greater consistency within their group than others, (e.g. respondents 3 and

249 10), when measuring the finished mouthguards at the anterior region point (Figures $3 \&$

250 4). At the other end of the consistency spectrum, there were technicians with high

251 variability in mouthguard manufacturing whilst using the same model, material and

252 machine. A case in point was respondent 11 who showed a $63 \%$ thickness variation,

253 Figure 4. The mean thickness of the mouthguards, from all samples, in the anterior 254 region was $1.62 \pm 0.38 \mathrm{~mm}$ with a range of $0.77-2.80 \mathrm{~mm}$ at the chosen specific single 
255 point on the anterior region. The reduction in thickness on forming from 4 to $0.77 \mathrm{~mm}$,

256 in the most extreme case, represents a total of $81 \%$ thinning of the material. From this

257 cohort of technicians, $52 \%$ had a greater material thinning than $1.62 \mathrm{~mm}$ at the anterior

258 region measurement point, with the mean thickness equating to an overall thinning of

$25959.5 \%$ in a single $4 \mathrm{~mm}$ laminate blank.

261 CT scanned comparison of two surfaces of the finished mouthguards

262 The scanned images act as a visual assessment tool for the thickness patterns observed 263 over the whole of the finished mouthguard, not just at the single pre-selected

264 measurement points. The blue/green colour denotes $1 \mathrm{~mm}+/-$ and the orange/red colour

265 denotes 3-4 $\mathrm{mm}$ as shown on the measurement range bar at the bottom of each image

266 (Figure 7).

267 In Figure 7 the first set of images show an example of the thinnest anterior labial flange 268 of the mouthguard which is shown in green, denoting the material has thinned to less

269 than $2 \mathrm{~mm}$, which concurs with the previous gauge measurements. The section labial to

270 the anterior teeth in most of the anterior view is shown as yellow, indicating the material

271 is $2 \mathrm{~mm}$ or above. Below this set of images is an example of the thickest mouthguard,

272 there was a marked colour change towards the yellow to red spectrum in the anterior

273 region, showing the mouthguard thickness increasing towards $3 \mathrm{~mm}$ around the anterior

274 teeth. The occlusal surface of this second set of images has a greater proliferation of red

275 and darker (Black) sections, where it forms into the deeper fissures of the posterior

276 teeth, indicating the mouthguard is thicker in this section also. 


\section{DISCUSSION}

281 The main focus of the current study was to investigate consistency in the thermoforming

282 procedure in relation to dimensional characteristics. We also aimed to ascertain which

283 parameters would be associated with decreased reproducibility in the thermoforming procedures

284 be they machine- or human-related. Thinning appears to be a consequence of the stretching of

285 the material into the extremities and undercuts areas of the model i.e. anterior sulcus and palatal

286 section. The current study showed $52 \%$ of the 100 mouthguards had a greater material thinning

287 of $1.62 \mathrm{~mm}$ within the anterior region point, with the mean thickness equating to an overall

288 thinning of $59.5 \%$ in a single $4 \mathrm{~mm}$ laminate blank. Excessive thinning may be addressed by the

289 use of a lamination technique, whereby two or more layers of mouthguard materials are bonded

290 together to create a thicker finished blank, with the aim of absorbing greater energy $(8,17)$. Our

291 study showed that the majority (i.e. 75\%) of the participant technicians do not laminate the

292 mouthguard material to increase the finished thickness. Westerman, Stringfellow, Eccleston (8)

293 found that a $1 \& 2 \mathrm{~mm}$ thickness of EVA offered lower protection in relation to energy

294 absorption when tested in the laboratory. Indeed, they reported that with $2 \mathrm{~mm}$, transmitting

$29515.70 \mathrm{kN}$, this was more than three times less effective as the $4 \mathrm{~mm}$ material, that transmitted

296 only $4.38 \mathrm{kN}$. The same study observed that there was only a marginal increase in material

297 performance, i.e. force transmission, through increasing the material thickness beyond $4 \mathrm{~mm}$,

298 with 5 and $6 \mathrm{~mm}$ blanks reducing transmission forces to $4.03 \mathrm{kN}$ and $3.91 \mathrm{kN}$ respectively (8).

299 Reductions in material thickness could increase injury risk if sports-induced impacts occurred

300 above the values stated by Westerman et al. (8). However, as they stated comfort is also an

301 issue within thicker guards thus a guard that is comfortable is better than not wearing one. Our

302 results showed notable inconsistencies/variations in the fabrication which agrees with previous

303 studies in terms of thinning, in particular with respect to thickness, in the anterior sulcus region

304 measurement point, both within and between individuals as shown in Figures 3 \& 4. The

305 greatest degree of material thinning and thickness inconsistency was observed in the anterior

306 sulcus region point of the finished mouthguard as shown in Figure 7. The occlusal and posterior 
307 lingual regions were much less of a problem. This study found there was an $81 \%$ thinning of the

308 processed mouthguard material in the most extreme case, from 4 to $0.77 \mathrm{~mm}$. This degree of

309 thinning is marginally higher than that described in the study by Geary et al. (4) in which a

310 thinning of $72 \%$ when using a $3 \mathrm{~mm}$ mouthguard blank was reported. The mean thickness of the

311 mouthguards, from all samples, in the anterior region, was $1.62 \pm 0.38 \mathrm{~mm}$ with a range of 0.77 -

$3122.8 \mathrm{~mm}$. At $1.62 \pm 0.38 \mathrm{~mm}$ the mean degree of thinning would be $59.5 \%$ of the original blank

313 thickness of $4 \mathrm{~mm}$, which is similar to studies by Del Rossi et al. (5) who reported thinning as

314 high as $60 \%$ in the labial surface of the incisal and canine dentition, when using $3 \mathrm{~mm}$

315 mouthguard blanks. Geary et al. (4) reported thinning in the anterior labial sub gingival region

316 of $49 \%$, which is a comparable measurement point to the anterior site as used in this study.

317 They also recorded thinning as high as $72 \%$ in the incisal region, also using $3 \mathrm{~mm}$ blanks (4).

318 However, within our study, 4 mm blanks were used showing that even the thicker blanks still

319 have significant variations in thinning. This study showed that $70 \%$ use $4 \mathrm{~mm}$ blanks for their

320 mouthguards (which may be used for single/dual laminate use), this is in accord with the earlier

321 personal communication with dental material suppliers. Conversely, studies by Geary et al. (4)

322 and Del Rossi et al. (5) used 3mm blanks, Mizuhashi et al. $(9,10,12) 3.8 \mathrm{~mm}$ blanks and

323 Takahashi et al. (11) $4 \mathrm{~mm}$ blanks.

325 Factors that may affect mouthguard material thinning.

326 Another key novel aspect in the present study was the large sample size not only in

327 terms of individuals, but also the total number of formed mouthguards; with most

328 earlier studies only using one investigator to form the mouthguards $(4,5)$. In our study,

329 with participation from twenty dental technicians, our data is arguably representative of

330 dimensional changes during fabrication in a commercial environment rather than

331 laboratory research (hence controlled) environment. To add to which, the technicians

332 were requested to complete an accompanying questionnaire, which collected data on the

333 technician's own material thickness preference, the age/make/type of forming machine 
334 used, the technician's level of experience (in numbers of years in practice), which may

335 have had a bearing on the finished thickness of the mouthguards.

336 The age of the thermoforming machines ranged from 1-20 years. This is relevant as in

337 most cases the thermoforming machine uses a halogen heater to heat the blank and over

338 time, the heaters may become less efficient and may not heat the blank evenly. The

339 effects of heating is an important factor as it has been shown as a potential key element

340 in terms of fit of the mouthguard $(10,11)$. Pressure forming machines are shown to be

341 the most widely used to make mouthguards even though they are the most expensive to

342 purchase. Only two of the twenty-strong cohort used vacuum forming machines making

343 a true statistical comparison unfeasible with regard to a comparison between vacuum

344 and pressure forming machines. It would have been ideal to make comparisons between

345 the vacuum forming types of machines. However, due to the study being blind we

346 cannot address which type was more favourable. To add to which, the human factor (i.e.

347 an individual's own technique) may have also been present during fabrication, and thus

348 difficut to separate from the true merits of the equipment. Nonetheless, we propose that

349 a direct comparison of makes of equipment could be a useful follow-up study to the

350 present research work. Statistically there were no correlations between the thickness of

351 the finished mouthguard and either the years of experience of the technician or the age,

352 make or type of machine used. Accepting this, other possible reasons for the observed

353 discrepancies within groups could be: different positioning of the models i.e.

354 orientation, as discussed previously in a study by Geary et al. (4), and/or distance from

355 the heat source, fluctuations in environmental temperature i.e. open window cooling the

356 blank or not following the manufacturer's instructions.

357 The level of experience of technicians ranged from 6-10 to 30+ years; arguably it might

358 have been assumed that with greater experience one would have seen less variation, in 
359 terms both of thickness and consistency. Interestingly, statistically there was no

360 significant difference $(\mathrm{p}>0.05)$ between the levels of experience of the groups of

361 technicians in this task. We therefore propose that the reason for this lack of influence of

362 number of year's-experience on the technician's results may be that different technicians

363 will allow the material to heat for indeterminate amounts of time. Consequently, since

364 the amount of time heating correlates to the degree of sag (amount the heated blank is

365 allowed to slump), ultimately this will have impacted on the degree of thinning of the

366 material prior to forming. The present study showed $70 \%$ of respondents used $4 \mathrm{~mm}$

367 mouthguard blanks for construction of their custom-made mouthguards. Following the

368 inherent thinning observed in the processing of the given cohort $52 \%$ had a greater

369 material thinning of $1.62 \mathrm{~mm}$ within the anterior region point and an overall thinning of

$37059.5 \%$ in a single $4 \mathrm{~mm}$ laminate blank. All in all the present study shows the

371 differences in consistency within, and between, groups of technicians in the fabrication

372 of this custom made mouthguard (Figures $3 \& 4$ ). 


\section{CONCLUSION}

374 The current production methods showed $52 \%$ of produced mouthguards had a material

375 thinning greater than $1.62 \mathrm{~mm}$ within the anterior region point, and an overall thinning 376 of $59.5 \%$ for a single laminate $4 \mathrm{~mm}$ mouthguard blank, at the chosen point at the

377 anterior sulcus, irrespective of the technicians level of experience or type/age of the

378 thermo-forming machine. It is recommended that prior to any mouthguard being sent to

379 the dentist, it should be measured in the thinnest section of key anatomical points, i.e.

380 anterior sulcus. The dental technology community also needs to be aware of these

381 issues in relation to the thermoforming technique, and not take it at face value that the

382 mouthguard's thickness would be consistent throughout the manufacturing process. This

383 recommendation applies regardless of the initial thickness of the blank as there are

384 variations within the degree of thinning. Differences in thickness may affect the

385 performance of the guard, particularly if a large impact was to occur during sports

386 activity $(8,13,18)$. However, it must be emphasised here that any form of custom made

387 mouthguard protection regardless of thickness is better than wearing none at all even

388 with the lower levels of thickness reported in this study $(7,19,20)$. Also, as

389 previously suggested by Patrick, van Noort, Found (18), a grading, based in part on the

390 thickness of the finished mouthguard whether by lamination, design or blank selection,

391 could be awarded to the mouthguard as to the level of protection the mouthguard affords

392 to an individual's chosen sport. This study highlights, the need for a definitive and

393 readily available guide for both the dentists and members of the public, to show the

394 correct thickness of mouthguard, so that an informed decision as to the adequacy of the

395 mouthguard to perform the expected function in relation to the selected sport. 


\section{References}

397 1. Yamada J, Maeda Y. Thermoforming process for fabricating oral appliances:

398 influence of heating and pressure application timing on formability. J Prosthodont. 2007

399 Nov-Dec; 16(6):452-56.

400 2. Patrick D G, van Noort R, Found M S. The influence of heat treatment on the

401 impact performance of sports mouthguard materials. Composites Part A: Applied 402 Science and Manufacturing. 2006; 37 (9):1423-27.

403 3. American Dental Association. Using mouthguards to reduce the incidence and 404 severity of sports-related oral injuries. J Am Dent Assoc. 2006 Dec;137 (12):1712-20.

405 4. Geary J L, Kinirons M J. Post thermoforming dimensional changes of ethylene 406 vinyl acetate used in custom-made mouthguards for trauma prevention--a pilot study. 407 Dent Traumatol. 2008 Jun; 24 (3):350-55.

408 5. Del Rossi G, Leyte-Vidal MA. Fabricating a better mouthguard. Part I: factors 409 influencing mouthguard thinning. Dent Traumatol. 2007 Jun; 23 (3):149-54.

410 6. Westerman B, Stringfellow P M, Eccleston J A. Forces transmitted through EVA 411 mouthguard materials of different types and thickness. Aust Dent J. 1995. 40(6), 389412391.

413 7. Maeda Y, Kumamoto D, Yagi K, Ikebe K. Effectiveness and fabrication of 414 mouthguards. Dent Traumatol. 2009 Dec;25 (6):556-564.

415 8. Westerman B, Stringfellow P M, Eccleston J A. EVA mouthguards: how thick 416 should they be? Dent Traumatol. 2002 Feb; 18(1):24-27.

417 9. Mizuhashi F, Koide K, Takahashi M. Thickness and fit of mouthguards 418 according to a vacuum-forming process. Dent Traumatol. 2013;29(4):307419312.

420 10. Mizuhashi F, Koide K, Takahashi M. Thickness and fit of mouthguards 421 according to heating methods. Dent Traumatol. 2014;30(1):60-64. 
422 11. Takahashi M, Koide K, Mizuhashi F. Variation in mouthguard thickness 423 due to different heating conditions during fabrication. J Prosthodont Res. 424 2013;57(3):179-85.

425 12. Mizuhashi F, Koide K, Takahashi M, Mizuhashi R. A method to maintain 426 the thickness of the mouthguard after the vacuum forming process: changes 427 of the holding conditions of the mouthguard sheet. Dent Traumatol. 428 2012;28: 291-295.

429 13. Tran D, Cooke M S, Newsome P R. Laboratory evaluation of 430 mouthguard material. Dent Traumatol. 2001 Dec;17 (6):260-65.

431 14. Wicks R A, Coco S, Ahuja S. A mouthguard fabrication technique for 432 contemporary sports dentistry. J Tenn Dent Assoc. 2009 Fall; 89(4):32-33.

433 15. Knuth A D. The art of computer programming. Fundamental algorithms $3^{\text {rd }}$ ed. Reading, 434 Massachusetts, Addison Wesley, 1997.

435 16. Anusavice K J, Phillips R W. Phillips' science of dental materials, St. Louis, 436 Mo; [Great Britain], Saunders. 2003; 231-238.

437 17. Patrick D G, van Noort R, Found M S. Evaluation of laminated structures for 438 sports mouthguards: Key Engineering Materials. 2002; 221-222:133-144.

439 18. Patrick D G, van Noort R, Found MS. Scale of protection and the various types 440 of sports mouthguard. Br J Sports Med. 2005 May;39 (5):278-81.

441 19. Ozawa T, Takeda T, Ishigami K, Narimatsu K, Hasegawa K, Nakajima K, 442 Noh K. Shock absorption ability of mouthguard against forceful, traumatic 443 mandibular closure. Dent Traumatol. 2014; 30:204-210.

444 20. Hoffmann J, Alfter G, Rudolph N K, Goz G. Experimental comparative study of 445 various mouthguards. Endod Dent Traumatol, 1999; 15(4):157-163. 